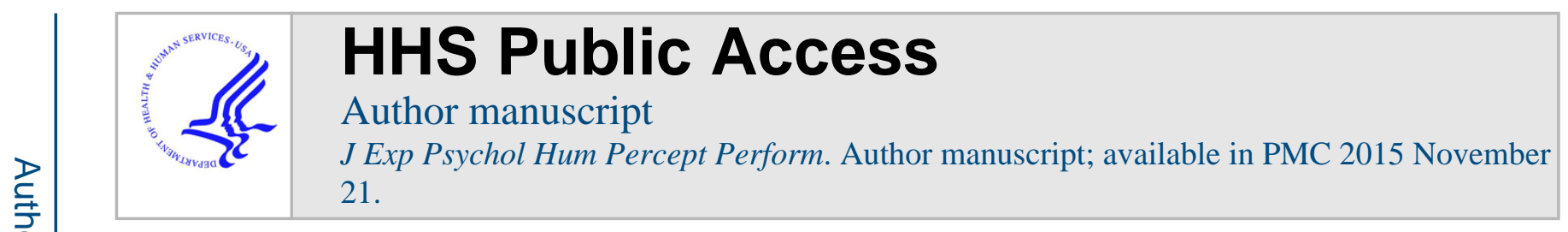

Published in final edited form as:

J Exp Psychol Hum Percept Perform. 2009 October ; 35(5): 1385-1397. doi:10.1037/a0016470.

\title{
Long-term, Abstract Learning of Attentional Set
}

\author{
Andrew B. Leber ${ }^{1}$, Jun-ichiro Kawahara ${ }^{2}$, and Yuji Gabari ${ }^{3}$ \\ ${ }^{1}$ University of New Hampshire \\ ${ }^{2}$ National Institute of Advanced Industrial Science and Technology \\ ${ }^{3}$ Hiroshima University
}

\begin{abstract}
How does past experience influence visual search strategy (i.e., attentional set)? Recent reports have shown that, when given the option to use 1 of 2 attentional sets, observers persist with the set previously required in a training phase. Here, we address 2 related questions. First, does the training effect result only from perseveration with the currently active set or from long-term learning? Experiment 1 supported the latter alternative: when training and test were separated by up to 1 week, to prevent perseveration across the 2 sessions, the training effect still obtained. Second, is the learning feature-specific (tuned to a precise set of colors) or more abstract? Experiments 2 and 3 supported the latter: when stimulus colors were switched between training and test to remove the possibility of feature-specific learning, the training effect again obtained. We conclude that attentional set is largely guided by long-term, abstract learning.
\end{abstract}

\section{Keywords}

Attentional set; learning; strategy; visual search; attention capture

When seeking to efficiently scan the visual world for an object of interest, we frequently have more than one suitable strategy at our disposal. Consider an example involving a soccer fan watching a game. Each time she glances away from the action (e.g., to talk with her friend, eat food, or drink beer), she must engage in a new visual search for the ball upon returning her eyes to the playing field. To quickly locate the ball, she may search for white things, round things, or perhaps objects on fast motion trajectories (for a review of effective search strategies, see Wolfe \& Horowitz, 2004). How is the selection of search strategy, or attentional set, made? Perhaps the most sensible approach would be to use the attentional set that best optimizes performance - that is, the one that allows detection or identification of the target most rapidly and accurately, while minimizing distraction from irrelevant information. However, countless research reports have exposed the use of non-optimal search behavior; that is, observers frequently have shown susceptibility to interference from irrelevant feature distractors (e.g., color singletons; see Theeuwes, 1991, 1992; Todd \&

Address correspondence to: Andrew Leber, Department of Psychology, University of New Hampshire, Conant Hall, 10 Library Way, Durham, NH 03824, 603.862.3170, andrew.leber@unh.edu.

Author Note

Yuji Gabari is now at the Application Platform Division, NEC System Technologies (NEC-ST), Hiroshima, Japan. 
Kramer, 1994; Turatto \& Galfano, 2001), although many reports have documented successful avoidance of such types of interference (see Bacon \& Egeth, 1994; Folk, Remington \& Johnston, 1992; Leber \& Egeth, 2006b). Given that the presumed optimal attentional set is not always used, other factors must play a role.

Recently, a number of studies have generated evidence supporting a significant role of past experience in the use of attentional set (Kawahara \& Gabari, 2006; Leber \& Egeth, 2006a, 2006b; Thompson, Underwood \& Crundall, 2007). Such studies have been motivated by the basic notion that associative learning pairs task sets with the environmental contexts in which they are used (see Norman \& Shallice, 1986; Shallice, 1988; see also Logan 1988); after extended use of a task set or action "schema" in one context (e.g., the soccer stadium or perhaps a testing room), future encounters with that context will trigger the automatic activation of that task set or schema. In the realm of visual attention, studies of contextual cueing have revealed that sufficient exposure to a visual search context serve to guide attentional orienting in subsequent encounters of the same context (Chun \& Jiang, 1998; Chun, 2000).

More specifically, regarding the current discussion of feature-based attentional set, one might predict that sufficient experience in using a set in a one context should bias the likelihood that it will again be used in the same context. Leber and Egeth (2006a) first produced data supporting this prediction, in an study in which they presented observers with a visual search task that could be solved by using one of two types of attentional sets. Observers searched a rapid serial visual presentation (RSVP) of non-target gray letters for a single non-gray target letter on each trial that was consistent in color across the block of trials (e.g., always red). On the one hand, because of the target's consistent color, the observers could adopt a narrow set for the specific target feature (feature search mode; see Bacon \& Egeth, 1994). On the other hand because the target was a color oddball, or singleton, with respect to its temporal neighbors, observers also could adopt a broad set tuned to salience - such that any singleton would gain processing priority (singleton detection mode; see Bacon \& Egeth, 1994; Pashler, 1988). Since more than one sufficient strategy was available to observers, these trials are referred to as option trials.

To manipulate past experience, Leber and Egeth preceded the option trials with a training phase designed to coax observers into either a singleton detection mode or a feature search mode (see Figure 1). Those assigned to the Feature group were asked to search for a consistently colored target (i.e., the same color as the forthcoming option trial targets) among heterogeneously colored non-targets in the RSVP stream. Here, because of the nontarget heterogeneity, the target was not a singleton, and thus a singleton detection mode would be unsatisfactory; rather, only a feature search mode would allow the observers to find the target. In contrast, those assigned to the Singleton group were asked to search for a target of unknown color (selected randomly from a set of 5 on each trial), which was embedded in a stream of homogeneously colored non-targets (all gray). Here, the target was always a singleton with respect to its homogeneous temporal neighbors, but its specific feature was unpredictable; because it was not possible to search for specific features, only a singleton detection mode could be used. While the two groups of observers would have to implement divergent attentional sets during this training phase, the critical question was 
whether they would carry these divergent sets into - and throughout - the test phase of option trials, which immediately followed training.

Results were consistent with the prediction that attentional set used on the option trials of the test phase - as indexed by sensitivity to irrelevant peripheral distractors (see Folk, Leber \& Egeth, 2002) - did indeed differ across groups. That is, those assigned to the Feature group for training persisted in using a feature search mode throughout the entire test phase, while those assigned to the Singleton group persisted in using singleton detection mode throughout the test phase. These data confirmed that past experience could exert a profound, lasting influence on the use of attentional set, and Leber and Egeth (2006a) concluded that associations formed between the environmental context and the chosen set during the training phase served to bias the use of set during the test phase. Similar results corroborating this conclusion have also been reported (Kawahara \& Gabari, 2006; Leber \& Egeth, 2006b; Thompson, Underwood, \& Crundall, 2007).

The main purpose of this article is to scrutinize the training effects under a more critical lens. The test phase results have been interpreted to arise from an associative pairing of the training context with the attentional set used - which in turn automatically biases the subsequent use of attentional set, as discussed above. However, we identify and address a potentially serious challenge to this learning account in the first part of this article (see Objective 1, below). In the second part, we further probe how past experience could steer observers into one attentional set or another (see Objective 2).

\section{Objective 1: Ruling out a "perseveration bias" account}

Beginning with the challenge, we raise the possibility that the training effects of the previous studies were not brought about by learning. Rather, an alternative that we refer to as the perseveration bias account holds that observers are naturally predisposed to continue using their currently active attentional set. According to this account, observers in the previous studies entered a very stable state of configuration to their attentional set over the course of roughly 30 minutes of training; then, owing to this deep state of configuration, the "path of least resistance" dictated that they maintain their currently configured state. This is distinct from the learning account in that the latter assumes that attentional set could be reactivated by the experimental context during test, rather than simply being maintained - without interruption - from training to test.

Ample evidence exists to support the possibility of a perseveration bias in the use of attentional set. Consulting the patient literature from which we borrowed the term, perseveration associated with frontal lobe damage is characterized by pathological "stuck in set" behavior after an initial task is configured (Luria, 1965; Milner, 1964; Sandson \& Albert, 1984). This behavior demonstrates an underlying tendency to persist with task sets, revealed when frontal lobe function is compromised. In normal populations, studies of task switching expose the difficulty of reconfiguring task set via unavoidable behavioral slowing (and sometimes accuracy decrements) when switching tasks compared to when repeating them (e.g., Allport, Styles, \& Hsieh, 1994; Jersild, 1927; Rogers \& Monsell, 1995). ${ }^{\mathrm{i}}$ More specific to the current discussion, costs of switching attentional set have also been well 
described (Hillstrom, 2000; Leber, 2004, Vickery, King, \& Jiang, 2005; Wolfe, Horowitz, Kenner, Hyle, \& Vassan, 2004). Lastly, studies of "voluntary task switching," in which participants are asked to alternate between two tasks at random (with the constraint that they switch on approximately half of the trials), consistently report a strong bias toward maintaining the previous task set (Arrington \& Logan, 2004, 2005; Mayr \& Bell, 2006). This latter finding shows that recent task sets are not only difficult to discard; observers attempt to maintain them whenever possible.

The possibility of a perseveration bias presents a challenge for the learning account because it does not assume or require any long-term association. Leber and Egeth (2006a) acknowledged the potential for the perseveration bias but attempted to rule it out based on the results of a second experiment, in which the training phase was reduced to 40 trials and the training effect did not arise. That is, the Feature and Singleton groups were using divergent attentional sets by the end of training, but they did not persist with these sets into test - a result that seems at odds with a perseveration bias. However, it is possible that observers were only weakly configured to their training-phase set by the time the option trials of the test phase were introduced. That is, assuming the stability of configuration to a current task set should increase along with the duration for which it is used, the likelihood of perseverating should also increase with time. Thus, it appears that the perseveration bias remains quite plausible, and in light of this, it is arguably still unknown whether long-term learning (e.g., the association between context and attentional set) can serve to guide the use of attentional set.

In seeking to effectively disentangle the learning account from the perseveration bias account, it is essential to consider that perseveration effects in patients are short-lived, lasting no more than one- or two-dozen trials (Cohen \& Dehaene, 1998), and the cost of switching from one task set to another also subsides over a similarly brief timecourse (e.g., Allport et al., 1994). Thus, once participants complete a training session (e.g., in the study of Leber \& Egeth, 2006a), leave the testing room, and tackle new visual search challenges on their paths home, the activation level for the current set should decay and any perseverative bias toward the recent set should abate. Any lasting effects of training, consequently, could only be the result of learning in such a scenario. Experiment 1 of the present study addresses this matter by inserting a delay of one day or one week between training and test, so that observers could return for the test session after the activation for the set used during training has presumably decayed. To preview the results, the effect of past experience was still observed, leading us to conclude that learning does indeed guide the use of attentional set.

\section{Objective 2: Providing evidence for abstract learning}

Upon confirming that learning occurs, we established our second objective in this study, which questioned exactly what kind of learning occurs. On the one hand, observers might be biased to use attentional sets such as singleton detection or feature search modes as a result of their training experience. On the other hand, it is possible that a lower-level type of

\footnotetext{
${ }^{\mathrm{i}}$ Although, note that even seemingly short-term, perseverative tendencies in task switching have been shown to be at least partially explained by long-term learning of stimuli that are specific to the particular task (Waszak et al., 2003).
} 
learning - what we refer to as feature-specific learning - is at work. Consider that in the previous studies of past experience (e.g., Leber \& Egeth, 2006a), observers in the Feature group spent the entire training phase searching for a single color - say, red. By the end of training, it is possible that they became more skilled at detecting red items. Similarly, observers in the Singleton group spent the entire training phase searching all-gray streams for singletons; it is possible that these observers became more skilled at ignoring gray items.

Improvement due to practice, particularly when low-level properties of the stimuli are consistent across experimental sessions, has long been documented in visual search performance (e.g., Ahissar \& Hochstein, 1993; Sagi \& Karni, 1992; Schneider \& Shiffrin, 1977; Shiffrin \& Schneider, 1977). Recently, Tseng, Gobell, and Sperling (2004) reported a particularly relevant study, in which they had observers search for 4-7 hours for a consistent target color in spatial arrays of items. Weeks later, Tseng et al. exposed the same observers to a third-order motion display, where motion could be carried in one direction via the previously searched for color or in another direction by a different color. The authors found their observers had become significantly more likely to perceive motion to be carried by the trained color, and they interpreted their data as evidence that the observers became attentionally "sensitized" to the trained color, which served to fundamentally alter that color's perceived salience. Note that observers in the studies we discussed previously completed only about 30 minutes of training (e.g., Kawahara \& Gabari, 2006; Leber \& Egeth, 2006a, 2006b), compared to 4-7 hours for the Tseng et al. study. Nevertheless, the kind of feature-specific learning they report provides a very plausible alternative to the higher-level "attentional set" account, which holds that observers learned something more abstract - namely, a mode of search (feature search or singleton detection mode), which need not be tied to the specific low-level properties of the stimulus displays. In Experiments 2 and 3, we control for the potential contribution of feature-specific learning to determine whether the abstract learning of attentional set can indeed occur, by changing the stimulus sets between training and test. To preview these results, the learning effect was still observed, supporting the abstract learning account.

\section{Experiment 1}

This experiment was designed to remove any possible contribution of a perseveration bias and then determine whether the effect of past experience would still obtain. Our approach was to introduce delays of one day or one week between training and test. To be consistent with previous work, we adopted the "option trial" design used by Leber and Egeth (2006a; see Figure 1).

As mentioned above, the Leber and Egeth study used RSVP streams where target and nontarget colors were manipulated during training to coax different attentional sets that would transfer into the test trials. To assess the use of attentional set, Leber and Egeth incorporated a distractor interference procedure introduced by Folk, Leber, and Egeth (2002; see Figure 2). In this procedure, a single frame of the RSVP stream on each trial could be accompanied by a peripheral distractor display, which contained four pound signs (i.e., "\#”) that were placed approximately 5 degrees of visual angle above, below, to the left, and to the right of fixation. In the "all gray" condition, the four distractors were colored gray. In the "same 
singleton" condition, three distractors were gray, while the fourth was matched to the color of the target (red or green). The "different singleton" condition also contained three gray distractors, but the fourth was a non-target color (green or red, depending on the observer's target color). Additionally, some trials contained no distractors. In this paradigm, significant interference is typically observed when the distractor display contains an item matching the attentional set of the observer (i.e., "contingent capture"; Folk, Remington, \& Johnston, 1992; Folk \& Remington, 1998), with interference peaking when the distractors precede the target by more than 150 milliseconds (ms) (Folk et al., 2002; Lamy, Leber \& Egeth, 2004; Leblanc \& Jolicoeur, 2005).

For the current purposes, we capitalized on the fact that the pattern of interference produced by the peripheral distractors should index the type of attentional set observers use on the option trials of the test phase. For example, observers using a feature search mode should only be susceptible to interference from the "same singleton" distractor display, and not the "different singleton" display, since the peripheral singleton item would not match the observer's attentional set. However, observers using a singleton detection mode should be vulnerable to distraction by any salient information, and would thus suffer interference from both same and different singleton displays.

We predicted that if long-term learning spans the gap of 1-day and 1-week delays between training and test, the patterns of distractor interference in the test session should differ between Feature and Singleton groups. Specifically, those in the Feature group should experience interference by only the same singleton while those in the singleton group should experience interference by both same and different singletons. Such divergent use of attentional set would only be attributable to learning, and not a perseveration bias, because upon finishing the training session and leaving the laboratory, observers would need to abandon the attentional sets they acquired during training in order to meet the many visual search challenges of everyday life. Given that participants would clearly not perseverate with, say, searching for red for an entire day or week, an effect of past experience should occur only if the context of the testing environment served to trigger the reactivation of the attentional set used during training.

\section{Method}

\section{Participants}

28 Hiroshima University undergraduates participated in the one-day delay treatment while 30 undergraduates participated in the one-week delay treatment. Each participant reported normal or corrected-to-normal visual acuity and normal color vision.

\section{Materials}

Stimuli were generated with a personal computer, using Matlab (Mathworks, Natick, MA), with the Psychophysics Toolbox extensions (Brainard, 1997; Pelli, 1997). Displays were presented on a 17 inch VGA monitor and viewed at an average distance of $50 \mathrm{~cm}$ (head position was not fixed). RSVP stream stimuli were drawn from the English alphabet (excluding I, O, Q, and Z), using Courier New font, with letters subtending $1.0^{\circ}$ in height by 
up to $1.0^{\circ}$ in width with a stroke of $0.09^{\circ}$. Stream presentations, which were centered at fixation, consisted of a 500 millisecond presentation of a white fixation cross $\left(0.4^{\circ}\right.$ tall by $0.4^{\circ}$ wide, stroke $=0.05^{\circ}$ ), followed by a $200 \mathrm{~ms}$ blank inter-stimulus interval (ISI). Then, 20 letters appeared in temporal succession, each selected randomly without replacement from the 22-letter set. Each letter was exposed for 50 milliseconds (ms), followed by a 50 ms blank, yielding a letter to letter stimulus onset asynchrony (SOA) of $100 \mathrm{~ms}$. Each letter, depending on variables described below, was gray, blue, purple, yellow, orange, green or red. Distractor displays, when present, entailed a $50 \mathrm{~ms}$ exposure of four pound signs (i.e., “\#”; $1.0^{\circ}$ tall, $1.0^{\circ}$ wide, stroke $=0.09^{\circ}$ ), which were centered $5.2^{\circ}$ above, below, to the right, and to the left of fixation; the temporal onset of these displays coincided with the onset of the stream letter appearing two serial positions prior to the target (i.e., $200 \mathrm{~ms}$ ). Distractor items were colored gray, red, or green, depending on variables described below. All stimuli were presented on a black background.

\section{Design}

Training Session-Within both 1-day and 1-week delay treatments, half of the observers were assigned to the Feature group while the other half were assigned to the Singleton group. The session lasted approximately 25 minutes.

Within each group (and for each delay treatment), color assignment, a variable that was used to determine the observer's target color, was counterbalanced between observers; half of the observers in each group were assigned "red" and the remaining observers were assigned "green."

Observers in the Feature group searched for a target of consistent color every trial (red or green, depending on color assignment), which was embedded in a stream of heterogeneously colored non-targets (gray, blue, purple, and green or red, depending on color assignment); the color of each non-target stream letter in these trials was selected randomly with replacement. Observers in the Singleton group searched for a target that on a given trial could be any one of five colors, selected randomly with replacement and unpredictable to observers. One color was determined by the observers' color assignment (red or green), while the remaining colors were purple, blue, yellow, and orange for all observers. The nontargets in the stream, for the Singleton group in this phase, were all homogeneous in color (gray) on every trial.

For both the Singleton and Feature groups, three independent variables were manipulated within observers to determine the stimulus characteristics on each trial: distractor type (4 levels), singleton location (4), and temporal target position (5). (The latter two variables were introduced for experimental control and collapsed across all levels for the analyses.) Crossing these variables yielded 80 unique conditions, which were each presented four times to generate 320 total trials, which were presented in random order. The variables are described as follows.

Distractor type: There were four distractor conditions, each presented equally often. In the "all-gray" condition, all four of the peripheral "\#” distractors were gray. In the "samecolored singleton" condition, three of the items were gray and the remaining one matched 
the observers' color assignment (red or green). The "different-colored singleton" condition contained three gray items and one nontarget-colored item (green or red, depending on the observers' color assignment). Lastly, there was a "no distractor" condition.

Singleton location: When distractor displays containing singletons were present, the singleton's spatial location was determined by this variable, ensuring that the singletons appeared equally often at the four peripheral locations.

Temporal target position: The target appeared simultaneously with one of 5 letters in the stream (serial positions 12 through 16, each with equal frequency).

Test Session-In this session, which lasted approximately 25 minutes, all observers were presented with "option" trials, where a consistently colored target was embedded in a stream of homogeneously colored (gray) distractors. The target color, determined by the color assignment variable, was the same in the test phase as it was for the training phase; for example, an observer assigned to the Feature group, who had searched for red targets in the training session, would continue to search for red targets in the test session.

Within-subject variables (distractor type, singleton location, and temporal target position) were manipulated in this session in the same fashion as in the training session, to yield 320 total trials, presented in random order.

\section{Procedure}

Participants were instructed to identify a color-defined target that was embedded in a rapid stream of letters at fixation. During the training session, Singleton group observers were instructed to search for the uniquely colored item in the RSVP stream, while Feature group observers were instructed to search for the red (or green) item in the stream. In the test session, all observers were informed that the target would be consistently colored (i.e., red or green, depending on color assignment) for the remainder of the experiment. All observers were asked to report the target's identity by entering the correct letter into a computer keyboard after the completion of the RSVP stream. Also, they were informed about the peripheral distractors and told to ignore them. Responses, which were collected at the end of each stream presentation, were unspeeded, and accuracy was emphasized. The session consisted of 24 practice trials, followed by the 320 trials of training. Depending on the observers' delay treatment, they returned one day or seven days later for the test session, where they completed 24 practice trials, followed by the 320 test trials. Practice trials prior to training and test were of the same type as the main trials of each respective session (i.e., feature or singleton search prior to training and "option" trials prior to test). During the main trials of both sessions, breaks were given every 40 trials.

Trials were initiated by a spacebar press, which prompted a blank-screen presentation for $1000 \mathrm{~ms}$, which was then followed by the RSVP stream presentation. At the completion of the stream, participants were prompted to report the target letter. A 250 ms feedback tone was presented for incorrect responses. 


\section{Results and Discussion: One-day Delay}

\section{Training Session}

Results of the training session confirmed that observers in the Feature and Singleton groups adopted the respective attentional sets intended by the experimental manipulations.

For the Feature group, mean accuracy scores in the four distractor conditions (none, all gray, same singleton, and different singleton) were $83.6 \%, 76.7 \%, 63.6 \%$, and $77.6 \%$, respectively, and a one-way ANOVA showed a significant effect of distractor condition, $F(3,39)=18.281, p<0.0001$. Comparisons between all combinations of pairs were carried out using Ryan's method (family-wise alpha $=0.05$; all uncorrected p-values associated with significant pairwise comparisons are reported). Results revealed that performance in the same singleton condition was worse than in the none, all gray, and different singleton conditions, all $p$ ' $s<0.0001$. No other comparisons were significant. This selective pattern of susceptibility to distraction by singletons matching the target color but not to equally salient non-matching singletons confirms that the Feature group observers used a feature search mode during training.

For the Singleton group, mean accuracy scores in the none, all gray, same singleton, and different singleton distractor conditions were $89.6 \%, 81.3 \%, 73.3 \%$, and $72.8 \%$, respectively, and the one-way ANOVA was significant, $F(3,39)=16.712, p<0.0001$. Pairwise comparisons showed that performance in both same and different singleton conditions was worse than in the no distractor, $p<0.0001$ and $p<0.0001$, respectively, as well as the all gray condition, $p<0.01$ and $p<0.005$, respectively, revealing impairment by both singletons; moreover, same and different conditions did not significantly differ from one another, suggesting equivalent impairment by both singletons. Additionally, performance in the all gray condition was worse than in the none condition, $p<0.005$, revealing some decrement here. Taken together, the pattern indicates that the Singleton group used a singleton detection mode during training.

\section{Test Session}

Data were first analyzed separately for the Feature and Singleton groups to characterize the attentional set used during this session.

Feature group accuracy scores, for which means are plotted in the left panel of Figure 3, were entered into a one-way ANOVA (across the 4 levels of distractor condition), which reached significance, $F(3,39)=14.689, p<0.0001$. As in training, pairwise comparisons showed that performance in the same singleton condition was worse than the none, $p<$ 0.0001 , all gray, $p<0.0001$, and different singleton conditions, $p<0.0005$, while no other comparisons were significant. This pattern indicates that the Feature group used a feature search mode in the test session.

For the Singleton group, for which means are also plotted in the left panel of Figure 3, the ANOVA was significant, $F(3,39)=23.645, p<0.0001$. Pairwise comparisons revealed that performance in both the same and different singleton conditions was worse than in the no distractor condition, both $p$ 's $<0.0001$, while these two conditions did not differ 
significantly from each other. This nonselective interference by the peripheral distractors indicates that the Singleton group used a singleton detection mode during the test session. Interestingly, the pairwise comparisons further revealed that the all gray condition was significantly worse than the no distractor condition, as it was in training, $p<0.0001-$ and so much so that the two singleton conditions did not yield worse performance than this condition. This may indicate that observers were so tuned for any type of salience that even the peripheral gray onsets produced considerable distraction.

To further test the hypothesis that past experience would lead to divergent use of attentional set in the test session, we compared performance across the groups. Specifically, we probed whether the performance in the same and different singletons would interact as a function of training-group assignment. To this end, we carried out a mixed model ANOVA, including the between-subjects factor of training group assignment ( 2 levels: Singleton and Feature groups) and the within-subjects factor of distractor condition (2 levels: same and different singleton).

Results revealed a main effect of distractor condition, $F(1,26)=9.708, p<0.005$, but no main effect of training group assignment, $F(1,26)=1.325$, n.s. Critically, the training group assignment $\times$ distractor condition interaction reached significance, $F(1,26)=5.981, p<$ 0.05 , indicating that the two groups of observers exhibited different usage of attentional set in the test session. That is, upon returning to the laboratory after 1 day of training, observers' performance was still influenced by the training session. We proceed to the one-week results before remarking upon this further.

\section{Results and Discussion: One-week Delay}

Two observers who completed the training session did not return for the test session, and they were thus excluded from this analysis.

\section{Training Session}

For the Feature group, mean accuracy scores in the four distractor conditions (none, all gray, same singleton, and different singleton) were $77.4 \%, 68.6 \%, 50.7 \%$, and $72.4 \%$, respectively, and the ANOVA was significant, $F(3,39)=25.059, \mathrm{p}<0.0001$. Pairwise comparisons showed that performance in the same singleton condition was worse than in the none, all gray, and different singleton conditions, all $p$ 's $<0.0001$. Additionally, the all gray distractor condition was significantly worse than the no distractor condition, $p<0.02$, but no other comparisons were significant. The selectively worse performance by the same singleton compared to the different singleton indicates that the Feature group observers used a feature search mode during training.

For the Singleton group, mean accuracy scores in the none, all gray, same singleton, and different singleton distractor conditions were $88.4 \%, 82.1 \%, 79.1 \%$, and $76.5 \%$, respectively, and the one-way ANOVA was significant, $F(3,39)=11.139, p<0.0001$. Pairwise comparisons showed that performance in both same and different singleton conditions was worse than in the no distractor condition, $p<0.0005$ and $p<0.0001$, respectively, revealing impairment by both singletons; moreover, same and different 
conditions did not significantly differ from one another, suggesting equivalent impairment by both singletons. This pattern indicates that a singleton detection mode was used.

Additionally, as was seen in the test session for the 1-day delay treatment, performance in the all gray condition was significantly worse than in the no distractor condition, $p<0.01$, while performance was not significantly worse for either of the singleton conditions than the all gray condition. Again, this may suggest that observers were susceptible to any type of salience in the display.

\section{Test Session}

Feature group accuracy means are plotted in the right panel of Figure 3. The one-way ANOVA was significant, $F(3,33)=14.801, p<0.0001$. As in training, pairwise comparisons showed that performance in the same singleton condition was worse than in the none, all gray, and different singleton conditions, all $p$ 's $<0.0001$, while no other comparisons were significant. This pattern indicates that the Feature group used a feature search mode in the test session.

Singleton group accuracy means are also plotted in the right panel of Figure 3, and the oneway ANOVA was also significant, $F(3,33)=16.258, p<0.0001$. Pairwise comparisons revealed that performance in both the same and different singleton conditions was worse than in the no distractor condition, both $p$ 's $<0.0001$, while the two conditions did not differ significantly from one another. This nonselective interference by the singleton distractors indicates that the Singleton group used a singleton detection mode during the test session. Additionally, the pairwise comparisons further showed that the all gray condition was significantly worse than the no distractor condition, $p<0.03$; however, this time both same and different singleton conditions did yield worse performance than the all gray condition, $p$ $<0.0005$ and $p<0.02$, respectively.

As with the one-day delay treatment, we carried out a training group assignment $\times$ singleton distractor condition mixed model ANOVA to determine whether performance during the test session differed significantly across groups. This analysis returned a main effect of distractor condition, $F(1,22)=18.457, p<0.0005$, but no main effect of training group assignment, $F$ $<1$. The critical training group $\times$ distractor condition interaction did reach significance, $F(1$, $22)=9.334, p<0.01$. Thus, as in the 1-day delay treatment, Singleton and Feature group observers who participated in the test session one week after training showed divergent patterns of distractor interference, reflecting divergent use of attentional set during the option trials. That is, participants who trained in the Singleton group returned after one week and used a singleton detection mode, while those who trained in the Feature group returned after one week and used a feature search mode.

\section{Discussion}

Observers who were exposed to identical conditions in the test phase showed dramatically different use of attentional set, depending on their experiences from just a 25 minute session that had occurred up to one week prior. These results cannot be explained by a perseveration bias, as perseveration decays over the course of minutes, not days (Allport et al., 1994; Cohen \& Dehaene, 1998); it would of course be especially maladaptive if the participants 
actively maintained their attentional sets for features or oddballs over the entire delay between training and test. In further contrast to the perseveration account, an inspection of Figure 3 shows that the training effect, rather than waning over the delay, appeared stronger at one-week than one-day. This pattern was not reliable, though; we ran a new 3-factor ANOVA (delay $\times$ training group assignment $\times$ singleton distractor condition) to determine whether delay modulated the training group assignment $\times$ singleton distractor condition interaction. The 3-way interaction was not significant, $F(1,48)<1$. Nevertheless, the numerical pattern is reminiscent of reports that contextual memory effects are more pronounced at one week than at shorter delays (Smith \& Vela, 2001).

All told, we attribute the long-term effects observed in this experiment to learning. Evidently, some aspect of the testing environment served to trigger a reactivation of the set that had been used during training. Given this confirmation of learning, we continue on to the next experiment, which examines more closely what kind of learning occurs.

\section{Experiment 2}

The approach in this experiment was to remove any possible contribution of feature-specific learning and then determine whether evidence for a more abstract form of learning would remain. Recall, from the introduction, our point that in previous studies of past experience and attentional set (e.g., Leber and Egeth, 2006a), observers in the Feature group might have improved at searching for red while observers in the Singleton group might have improved at ignoring gray. Thus, when reaching the test trials, observers might have continued with their previous sets because they were more perceptually skilled at those sets than the newly available alternative sets. As our goal was to remove any potential contribution of such feature-specific learning, we changed the stimuli as follows.

For the Feature group participants, their training phase was similar to before, where they searched for a consistent target color among heterogeneously colored nontargets. However, the value of this color changed in the test phase. Specifically, observers searching for red during training now searched for green during test, and those who searched for green during training now searched for red during test (whereas observers in the previous experiments would have continued to search for the same color). Since any experience with searching for, say, red during training would not confer a benefit upon searching for, say, green during test, the feature-specific learning account would not predict a tendency to use a feature search mode for the new target color. That is, there is no basis to predict that the now "same" singleton distractor will cause greater interference than the now "different" singleton distractor. Moreover, because this "different" singleton distractor matches the old target color (i.e., the one searched for in training), a feature-specific learning account might even predict that interference from this color should be magnified; in other words, it might be more difficult to ignore a feature that was previously the target. Indeed, Shiffrin and Schneider (1977) found exactly this phenomenon, albeit after thousands of trials of training prior to the remapping of target and distractor features in their search task. Nevertheless, despite training with too few trials to reasonably expect strong development of automaticity, a feature-specific learning account leans toward predicting that the old target color, which becomes a "different" singleton, will interfere as much or more as the new "same" singleton, 
which matches the present target color. In contrast to the predictions of the feature-specific learning account are the predictions of the abstract learning account, in which observers transfer their high-level search mode (i.e., feature search mode) to the test phase. This would manifest in the test phase via selective distraction of the now same-colored singleton, with minimal distraction from the now different-colored singleton.

Turning to the Singleton group, their training phase was much like that of previous studies, where the target color was selected randomly on each trial and embedded in a homogeneously-colored stream. However, now the stream color was a random non-target color that changed on each trial, rather than always being gray. Indeed, the stream color was never gray, so that observers had no exposure to gray during training. Then, in the option trials of the test phase, as in previous experiments, the non-target stream items became all gray (this was the same for the Feature group). Given that these observers would have no previous exposure to this non-target color, they would be unable to bring any advantage of suppressing this particular color into the test phase. As a result, the feature-specific learning account does not predict any special tendency toward using a singleton detection mode during test. However, if these observers carry the abstract mode of singleton detection into the test phase, there should be interference from both target-colored and nontarget-colored peripheral distractors.

Note that, like previous experiments, the key focus of the analysis was whether different search behavior across Feature and Singleton groups - as indexed by the patterns of distractor interference - would prevail in the identical conditions of the test phase option trials. As described above, for each particular group, only the abstract learning account predicts divergent patterns - that is, a tendency toward feature search mode for the Feature group and a tendency toward singleton detection mode for the Singleton group (although, recall the possibility that the feature-specific learning account might predict that the Feature group experience a magnified interference effect from the now different color singleton which, of course, would contrast with the predictions of the abstract learning account).

\section{Method}

\section{Participants}

Data were collected at the National Institute of Advanced Industrial Science and Technology (AIST), where 32 members of the local community were recruited to participate in exchange for monetary compensation. Each participant reported normal or corrected-to-normal visual acuity and normal color vision.

\section{Materials}

Stimuli were the same as in Experiment 1, with the following exceptions. Pilot data indicated that observers from this subject population were reaching ceiling-level performance in the test session. To remedy this, for the test session - but not for the training session - we implemented two changes to increase task difficulty. First, we reduced the exposure duration of each stream letter to $33 \mathrm{~ms}$, though we maintained the blank interval at $50 \mathrm{~ms}$, yielding a new letter-to-letter SOA of $83 \mathrm{~ms}$ (note that we kept the distractor display 
onset fixed at "lag 2," so the distractor-target SOA became $166 \mathrm{~ms}$ in this experiment). Second, we reduced the visibility of the target by superimposing a "pepper" mask on it: within the $1^{\circ} \times 1^{\circ}$ space in which the target letter was drawn, we randomly placed 20 small background-colored squares, which were each $0.09^{\circ} \times 0.09^{\circ}$ in size. This simultaneous mask was presented only with the target - not with the nontarget stream letters (nor with the peripheral distractors). Stream letters could be red, green, cyan, blue, purple, yellow, orange, or gray, and peripheral distractors could be red or green, as described below.

\section{Design}

Training Session-In this session, which lasted approximately 30 minutes, half of the observers were assigned to the Feature group while the remaining observers were assigned to the Singleton group. Within each of these groups, half of the observers had a "red" color assignment while the remaining observers were assigned "green."

Observers in the Feature group searched for a target of consistent color among heterogeneous nontargets (randomly chosen with replacement from the set of cyan, blue, purple, yellow, and orange on each frame). Observers in the Singleton group searched for a target that could be any one of 5 colors on a given trial, selected randomly with replacement and unpredictable to observers. None of these colors were red or green; rather, they were chosen randomly from the set of cyan, blue, purple, yellow, and orange. The nontargets for the singleton group were homogeneous in color on each trial, but never gray; rather, the nontarget color was selected randomly with replacement on each trial from the set of cyan, blue, purple, yellow, and orange, with the constraint that it differ from the target color.

Because one concern of the feature-specific learning account was that observers in the singleton group became skilled at ignoring gray during training, we took the additional step of removing gray items from the peripheral distractor displays. Our reasoning here was that observers might also attempt to suppress the irrelevant gray distractors - and thus gain some experience at ignoring this color, during training. Instead of substituting the gray items with another color, we chose to leave these locations blank. As a result, the same- and differentcolored singleton displays now only contained one peripheral item, which was red or green. ${ }^{\text {ii }}$ Furthermore, rather than replacing the all-gray display with another color, we simply omitted this distractor condition from the experiment, leaving 3 distractor types: none, same singleton, and different singleton. Note that for the Singleton group, because the observers did not view red or green targets during training, the condition labels "same singleton" and "different singleton" were essentially misnomers, and were "dummy coded" based on the observers' color assignment. Thus, while no performance differences were expected between these two conditions, they served to allow the examination of whether more than one peripheral color could produce interference.

\footnotetext{
ii One might argue that the peripheral distractors should no longer be labeled singletons, as they are presented alone. However, we do not feel that an object need be accompanied by spatially (or temporally) neighboring objects to be meet the literal definition of "singleton" (i.e., "one of a kind"); after all, the uniformly black background provides sufficient contrast to classify the peripheral distractors as unique.
} 
When crossing the 3 distractor types with 4 singleton locations and 5 temporal target positions (as used in Exp 1), 60 unique conditions were generated, which were each presented 6 times for a total of 360 trials.

Test Session-This session directly followed training and lasted approximately 30 minutes. As in Experiment 1, all observers were given "option" trials, with a consistently colored target appearing embedded in an all-gray RSVP stream. The target color in this session was switched from the initial color assignment value from the training session (i.e., red to green or green to red). That is, observers in the Feature group who initially searched for, say, green, were now asked to search for red (and vice-versa). The distractor conditions in this session were the same as those used during training (i.e., singleton distractor conditions consisted of a single red or green item in the periphery).

As with training, we crossed the same 3 distractor types with 4 singleton locations and 5 temporal target positions, and 360 total test trials were presented.

\section{Procedure}

The procedure was similar to Experiment 1, with the primary exception being that the test phase directly followed the training phase. Also, both training and test phases now had 360 main trials, as described above, and they were each preceded by 24 practice trials.

\section{Results and Discussion}

\section{Training Session}

For the Feature group, mean accuracy scores in the three distractor conditions (none, same singleton, and different singleton) were $65.9 \%, 51.3 \%$, and $62.2 \%$. A one-way ANOVA showed the main effect of distractor condition to be significant, $F(2,30)=22.435, p<$ 0.0001 . Pairwise comparisons revealed that performance was worse for the same singleton condition than both no distractor and different singleton conditions, both $p$ 's $<0.0001$, while these latter two conditions were not significantly different from one another. This pattern, reflecting selective interference by the same-colored singleton, indicates that the Feature group used a feature search mode during training.

For the Singleton group, mean accuracy values were $82.4 \%, 57.9 \%$, and $57.7 \%$ for none, same singleton, and different singleton conditions, respectively (for interpreting these data, recall that "same" and "different" conditions were functionally identical to one another, since neither singleton color matched any of the five target colors used for the Singleton group during training). The one-way ANOVA was significant, $F(2,30)=74.830, p<$ 0.0001 , and the pairwise comparisons showed this was driven by significantly worse performance both of the singleton conditions compared to the no distractor condition, both $p$ 's $<0.0001$; the two singleton conditions of course did not differ significantly. These results indicate that the Singleton group used a singleton detection mode during training.

\section{Test Session}

For the Feature group, mean accuracy scores are plotted in Figure 4. The one-way ANOVA reached significance, $F(2,30)=7.687, p<0.005$, and pairwise comparisons revealed this 
result to be driven by significantly worse performance in the same singleton condition compared to the no distractor and different distractor condition, both $p$ 's $<0.005$. The different distractor condition was not significantly different from the no-distractor condition. This pattern of data shows that Feature group observers used a feature search mode during test.

Mean accuracy scores for the Singleton group are also plotted in Figure 4. The one-way ANOVA reached significance, $F(2,30)=5.606, p<0.01$; pairwise comparisons showed that this result was driven by significantly worse performance in both same and different singleton conditions compared to the no distractor condition, $p<0.005$ and $p<0.03$, respectively, though the two singleton conditions were not significantly different from each other. These data indicate that Singleton group observers used a singleton detection mode during the test session.

As in the previous experiment, we wished to establish whether attentional set differed significantly across groups during the test session. Thus, we carried out a mixed model ANOVA, with training group assignment (Singleton and Feature groups) serving as the between-subjects factor and distractor condition (same and different singletons) serving as the within-subjects factor. This analysis yielded a main effect of distractor condition, $F(1$, $30)=17.245, p<0.0005$, but no main effect of training group assignment, $F<1$. The critical distractor $\times$ training group interaction reached significance, $F(1,30)=5.870, p<$ 0.05 , affirming that the impact of same and different singletons varied as a function of group assignment, and this reflected the divergent use of attentional set across groups.

Taken together, the results of this experiment are clear. Despite controlling for any potential contribution of feature-specific learning, the effect of past experience was still quite conspicuous. For the Singleton group, the entire set of target and nontarget colors was changed between training and test. Nevertheless, these observers carried forward their use of attentional set, as their behavior during the option trials confirms. For the Feature group, after completing training, these observers had to search the option trials in the test session for a new target color. Nevertheless, these observers performed in a manner quite indicative of feature search mode, as they only showed impairment to peripheral singleton distractors matching the new color. What is perhaps most remarkable about this result is the lack of impairment by the different singleton distractor during test - despite the fact that it now matched the old target color from the preceding training session. That is, an observer who initially searched for red and successfully ignored green during training was now searching for green and successfully ignoring red during test. We will revisit this observation in the General Discussion.

\section{Experiment 3}

This experiment was designed to address a potential limitation of Experiment 2 that relates to a different form of feature-based learning than what we initially considered. Rather than only improving at searching for and/or ignoring specific color values during training, it is also possible that observers gained proficiency at discriminating between sets of colors. For instance, if observers in the Feature group became more efficient in discriminating between red and green during training, then it would be easier for these individuals to separate red 
from green during test; thus, it might be easier to implement a feature search mode, despite the reversal of the color mappings. ${ }^{\text {iii }}$ In the present experiment, we addressed this issue by using completely different (i.e., non-overlapping) sets of colors in training and test. If the results of Experiment 2 were due to an improvement in discriminating between color values used during training (e.g., red/green), then the learning effect should no longer obtain when non-overlapping sets of colors are used between training and test. In contrast, if the results of Experiment 2 were not simply due to improvements in color discrimination, then the learning effect should still obtain (replicating the pattern of Experiment 2), despite the use of non-overlapping colors.

\section{Method}

\section{Participants}

Data were collected at the University of New Hampshire, where 56 undergraduates participated for course credit. Each participant reported normal or corrected-to-normal visual acuity and normal color vision.

\section{Materials}

Stimuli were generated with an Apple PowerMac and displayed on a 17" LCD monitor, again using Matlab (Mathworks, Natick, MA), with the Psychophysics Toolbox extensions (Brainard, 1997; Pelli, 1997). RSVP stream stimuli were drawn from the English alphabet (excluding I, M, Q, and Z), using Arial Bold font, with letters subtending $0.9^{\circ}$ in height by up to $0.9^{\circ}$ in width with a stroke of $0.2^{\circ}$. The fixation cross was $0.6^{\circ}$ tall by $0.6^{\circ}$ wide (stroke $=0.15^{\circ}$ ). Piloting was carried out to determine optimal stream presentation rates for keeping accuracy in a sensitive range for both training and test: for training, each letter was exposed for $67 \mathrm{~ms}$, followed by a $50 \mathrm{~ms}$ blank, yielding a letter to letter SOA of $117 \mathrm{~ms}$; for test, each letter was exposed for $50 \mathrm{~ms}$, followed by a $33 \mathrm{~ms}$ blank, yielding a letter to letter SOA of 83 ms. Unlike Experiment 2, simultaneous "pepper" masks were not used in this Experiment. Peripheral "\#” distractors were presented at an eccentricity of $4.6^{\circ}$. During training, stream letters could be cyan, purple, yellow, or orange, and peripheral distractors could be cyan or purple. During test, a new set of colors was used: stream letters could be gray, red, or green, and peripheral distractors could be red or green.

\section{Design}

Training Session-In the feature group, half of the observers searched for cyan targets and the other observers searched for purple targets. These targets were embedded among heterogeneous nontarget streams of yellow, orange, and either cyan or purple (whichever was not the target color). For observers in the Singleton group, the target color was selected randomly on each trial from the set of yellow, orange, cyan, and purple. From the remaining 3 colors of this set, the color of the homogeneous stream was randomly selected.

The independent variables were the same as in Experiment 2, with one modification: For the 3 distractor types, the no-distractor condition was presented $50 \%$ of the time, the same

iii We thank Anne Hillstrom for this suggestion. 
singleton condition was presented $25 \%$ of the time, and the different singleton condition was presented 25\% of the time. Note once again for the Singleton group (as in Experiment 2), the labels "same singleton" and "different singleton," which were dummy coded, served little descriptive purpose and should be interpreted only as a gauge of whether more than one peripheral distractor color can capture attention.

The 3 distractor types were crossed with the 4 singleton locations and 5 temporal target positions to obtain a minimum of 80 trials, each of which were presented 4 times in the training session for a total of 320 trials.

Test Session-As in the previous experiments, all observers were given "option" trials, with a consistently colored target (red or green) appearing embedded in an all-gray RSVP stream. For observers initially assigned to the Feature group, the target color during test was equally likely to be red or green, regardless of whether these observers previously searched for cyan or purple.

As with training, we crossed the 3 distractor types with 4 singleton locations and 5 temporal target positions, and 320 total test trials were presented.

\section{Procedure}

First, observers completed 24 practice trials, followed by the 320 trials of training. Next, the observers completed 24 practice trials, followed by the 320 test trials. During the main trials of both sessions, breaks were given every 80 trials.

Trial events were similar to those of Experiments 1 and 2, with slight changes noted as follows. On each trial, the fixation cross was displayed until observers pressed the spacebar. This prompted a blank-screen presentation for $750 \mathrm{~ms}$, which was then followed by the RSVP stream. After a response was registered, the word "correct" or "incorrect" was then displayed for $500 \mathrm{~ms}$ to provide feedback.

\section{Results and Discussion}

\section{Training Session}

For the Feature group, mean accuracy scores in the three distractor conditions (none, same singleton, and different singleton) were $83.4 \%, 67.9 \%$, and $81.6 \%$. A one-way ANOVA showed the main effect of distractor condition to be significant, $F(2,54)=34.405, p<$ 0.0001. Pairwise comparisons revealed that performance was worse for the same singleton condition than both no distractor and different singleton conditions, both $p$ 's $<0.0001$, while these latter two conditions were not significantly different from one another. This pattern, reflecting selective interference by the same-colored singleton, indicates that the Feature group used a feature search mode during training.

For the Singleton group, recall that "same" and "different" singletons were dummy coded and should thus only be interpreted to show whether more than one distractor color could capture attention. Mean accuracy scores were $89.8 \%, 73.5 \%$, and $75.2 \%$ for none, same singleton, and different singleton conditions, respectively (recall that same and different 
singletons. These conditions should simply be interpreted for evidence that more than one color can interfere with performance, indicating a singleton detection mode. As such, the one-way ANOVA was significant, $F(2,54)=26.370, p<0.0001$, and the pairwise comparisons showed this was driven by significantly worse performance both the same and different singleton conditions compared to the no distractor condition, both $p$ 's $<0.0001$; the same and different singleton conditions did not differ significantly.

\section{Test Session}

For the Feature group, mean accuracy scores are plotted in Figure 5. The one-way ANOVA reached significance, $F(2,54)=5.896, p<0.005$, and pairwise comparisons revealed this result to be driven by significantly worse performance in the same singleton condition compared to the no distractor and different distractor condition, both $p$ 's $<0.02$. The different distractor condition was not significantly different from the no-distractor condition. This pattern of data shows that Feature group observers used a feature search mode during test.

Mean accuracy scores for the Singleton group are also plotted in Figure 5. The one-way ANOVA reached significance, $F(2,54)=18.327, p<0.0001$; pairwise comparisons showed that this result was driven by significantly worse performance in both same and different singleton conditions compared to the no distractor condition, both $p$ 's $<0.0001$, though the two singleton conditions were not significantly different from each other. These data indicate that Singleton group observers used a singleton detection mode during the test session.

As in the previous experiments, we wished to establish whether attentional set differed significantly across groups during the test session. Thus, we carried out a mixed model ANOVA, with training group assignment (Singleton and Feature groups) serving as the between-subjects factor and distractor condition (same and different singletons) serving as the within-subjects factor. This analysis yielded a marginal main effect of distractor condition, $F(1,54)=3.100, p<0.1$, but no main effect of training group assignment, $F<1$. The critical distractor $\times$ training group interaction reached significance, $F(1,54)=5.210, p$ $<0.05$, affirming that the impact of same and different singletons varied as a function of group assignment, and this reflected the divergent use of set across groups.

The results of this experiment closely replicated those of Experiment 2. Despite using completely non-overlapping sets of colors in the two sessions of the experiment, the training effect obtained. That is, without a contribution from feature specific learning - either learning the specific color values or becoming more proficient at making particular color discriminations - the results are only attributable to an abstract, high-level learning of attentional set.

\section{General Discussion}

We began by asking what factors guide the use of attentional set. After briefly discussing some recent findings that have documented a role of past experience, we questioned whether such effects were due to learning or could be accounted for by a perseveration bias - that is, 
a tendency to persist with the currently active set. We designed the first experiment to resolve this question; after requiring observers to use one attentional set for approximately 25 minutes, we sent them home for 1 day or 1 week to allow the activation of their trained set to decay. When they returned, we presented them with "option" trials, which made available the use of more than one suitable set, and the observers reliably reactivated the set they had used during training. Given that the perseveration bias could not have accounted for these data (unless observers perseverated with, say, searching for red for an entire week straight), only learning could have served to guide the implementation of set. Moreover, the reliability of the results after a one-week delay stands as testament to the robustness of the learning.

Given the confirmation of learning, we next questioned what kind of learning occurs. Experiment 2 demonstrated that the effect of experience transferred across changes in the low-level features of the targets and nontargets, which removed any potential contribution of feature-specific learning. It is thus evident that the learning of attentional set was abstract in nature.

To further reflect upon what the results of this experiment mean, it is useful to consider the Singleton and Feature groups separately. For the Singleton group, the implications are fairly intuitive; these observers transferred the abstract mode of searching for salience from training to test, rather than simply learning to search for non-gray items. Given the original conception of singleton detection mode as a pure salience-driven search (see Pashler, 1988), this conclusion is straightforward.

What is more surprising is the result from the Feature group. These observers dramatically reversed the priority with which they treated red and green in the display. While searching specifically for red and searching specifically for green can both be considered instances of feature search mode, as they narrowly prioritize one feature to the exclusion of all others (see Bacon \& Egeth, 1994), there previously was no solid basis to assume any kinship between one specific feature search mode (e.g., for red) and another (e.g., for green). Given that no single configuration of attentional set could encompass the behavior exhibited during both training and test for this group, and further considering that there are costs for alternating between specific instantiations of feature search mode (Hillstrom, 2000; Leber, 2004; Vickery et al., 2005; Wolfe et al., 2004), it is quite intriguing that the transfer occurred. This result uncovers novel evidence that feature searches are represented as more similar to one another than they are to singleton detection mode. One speculation is that a higher level representational scheme of task sets places the various feature searches under one branch of a hierarchy, while placing singleton detection mode elsewhere.

One may speculate further, and consider that observers engaged in a feature search mode enter an entirely different cognitive state than those engaged in a singleton detection mode. Consider an individual working on a strict deadline, with a clear set of goals before him. $\mathrm{He}$ may navigate his office building in a very focused state, using attentional sets designed to acquire only the relevant information that is pertinent to his immediate goals; he may not even notice his colleagues in the hallway during this period. Note that while he is in this state, he will surely need to switch from one specific attentional set to another (e.g., search 
for the silver-colored keys in his office, then search for a manila folder in the conference room, etc.). Thus, while maintaining an overarching state of focus, he requires sequential usage of different feature search modes. In contrast, after meeting his goal, he can relax his attentional state to accept any salient information that he has not prespecified as relevant to an immediate goal - i.e., entering a singleton detection mode, where he is more likely to again say hello to a friend. Our preliminary efforts to explore this speculative account, however, have yet to garner support for it; in a shape-to-color transfer experiment, observers' search behavior during a test session of color option trials (like those of the current experiment) was uninfluenced by the requirement to search for specific shapes vs. oddball shapes during the training phase (Leber \& Kawahara, 2008). While further experiments of this kind are needed, it is possible that the learning effects of attentional set are restricted within single feature dimensions (e.g., color).

To conclude, the experiments here show that attentional set is subject to more than simply the demands of the current task, but rather determined in large part by experience. The effects of experience extend well past the dissipation of perseverative biases, and the manner in which the learning is encoded is represented in more abstract terms than low-level features. This study helps paint a more detailed picture of the well-integrated and deeply interactive mechanisms of learning, cognitive control, and attentional selection.

\section{Acknowledgments}

We thank Anne Hillstrom, Hermann Müller, \& Jan Theeuwes for very helpful comments and criticisms on an earlier version of this manuscript. This research was supported by a grant from the Japan Society for the Promotion of Science (17730433) to J.-I.K. and a National Institutes of Health postdoctoral fellowship (MH070115) to A.B.L.

\section{References}

Ahissar M, Hochstein S. Attentional control of early perceptual learning. Proceedings of the National Academy of Sciences. 1993; 90(12):5718-5722.

Allport, DA.; Styles, EA.; Hsieh, S. Shifting intentional set: Exploring the dynamic control of tasks. In: Umilta, C.; Moscovitch, M., editors. Attention and Performance XV. Cambridge, MA: MIT Press; 1994. p. 421-452.

Arrington CM, Logan GD. The cost of a voluntary task switch. Psychological Science. 2004; 15(9): 610. [PubMed: 15327632]

Arrington CM, Logan GD. Voluntary task switching: Chasing the elusive homunculus. Journal of Experimental Psychology. Learning, Memory, and Cognition. 2005; 31(4):683-702.

Bacon WF, Egeth HE. Overriding stimulus-driven attentional capture. Perception \& Psychophysics. 1994; 55(5):485-496. [PubMed: 8008550]

Brainard DH. The Psychophysics Toolbox. Spatial Vision. 1997; 10:433-436. [PubMed: 9176952]

Chun MM, Jiang Y. Contextual cueing: Implicit memory and learning of visual context guides spatial attention. Cognitive Psychology. 1998; 36:28-71. [PubMed: 9679076]

Chun MM. Contextual cueing of visual attention. Trends in Cognitive Sciences. 2000; 4:170-178. [PubMed: 10782102]

Cohen L, Dehaene S. Competition between past and present: Assessment and interpretation of verbal perseverations. Brain. 1998; 121:1641-1659. [PubMed: 9762954]

Folk CL, Leber AB, Egeth HE. Made you blink! Contingent attentional capture produces a spatial blink. Perception \& Psychophysics. 2002; 64(5):741-753. [PubMed: 12201333] 
Folk CL, Remington R. Selectivity in distraction by irrelevant featural singletons: evidence for two forms of attentional capture. Journal of Experimental Psychology: Human Perception \& Performance. 1998; 24(3):847-858. [PubMed: 9627420]

Folk CL, Remington RW, Johnston JC. Involuntary covert orienting is contingent on attentional control settings. Journal of Experimental Psychology: Human Perception \& Performance. 1992; 18(4):1030-1044. [PubMed: 1431742]

Hillstrom AP. Repetition effects in visual search. Perception \& Psychophysics. 2000; 62:800-817. [PubMed: 10883586]

Karni A, Sagi D. The time course of learning a visual skill. Nature. 1993; 365(6443):250-252. [PubMed: 8371779]

Kawahara, J.; Gabari, Y. Learning of attentional set transfers between spatial visual search and RSVP identification tasks; Poster presented at the annual meeting of the Psychonomic Society; Houston, TX. 2006 Nov.

Lamy D, Leber A, Egeth HE. Effects of task relevance and stimulus-driven salience in feature-search mode. Journal of Experimental Psychology: Human Perception \& Performance. 2004; 30(6):10191031. [PubMed: 15584812]

Leber, AB. PhD dissertation. The Johns Hopkins University; United States - Maryland: 2004. On the maintenance and switching of attentional set. Retrieved September 1, 2007, from ProQuest Digital Dissertations database (Publication No. AAT 3107532)

Leber AB, Egeth HE. Attention on autopilot: Past experience and attentional set. Visual Cognition. 2006a; 14(4-8):565-583.

Leber AB, Egeth HE. It's under control: Top-down search strategies can override attentional capture. Psychonomic Bulletin \& Review. 2006b; 13(1):132-138. [PubMed: 16724780]

Leber, AB.; Kawahara, J. Abstract learning of attentional set; Poster presented at the annual meeting of the Vision Sciences Society; Naples, FL. 2008 May.

Leblanc E, Jolicoeur P. The time course of the contingent spatial blink. Canadian Journal of Experimental Psychology. 2005; 59(2):124-131. [PubMed: 16035345]

Logan GD. Toward an instance theory of automatization. Psychological Review. 1988; 95:492-527.

Luria AR. Two kinds of motor perseveration in massive injury of the frontal lobes. Brain. 1965; 88:110. [PubMed: 14280275]

Mayr U, Bell T. On how to be unpredictable: evidence from the voluntary task-switching paradigm. Psychological Science. 2006; 17(9):774-780. [PubMed: 16984294]

Milner. 1964

Norman, DA.; Shallice, T. Attention to action: Willed and automatic control of behavior. In: Schwartz, GE.; Shapiro, D., editors. Consciousness and self-regulation: Advances in research. Vol. IV. New York: Plenum Press; 1986. p. 1-18.

Pashler H. Cross-dimensional interaction and texture segregation. Perception \& Psychophysics. 1988; 43(4):307-318. [PubMed: 3362658]

Pelli DG. The VideoToolbox software for visual psychophysics: Transforming numbers into movies. Spatial Vision. 1997; 10:437-442. [PubMed: 9176953]

Rogers RD, Monsell S. Costs of a predictable switch between simple cognitive tasks. Journal of Experimental Psychology: General. 1995; 124(2):207-231.

Sandson J, Albert ML. Varieties of perseveration. Neuropsychologia. 1984; 22(6):715-732. [PubMed: 6084826]

Schneider W, Shiffrin RM. Controlled and automatic human information processing: I. Detection, search and attention. Psychological Review. 1977; 84(1):1-66.

Shiffrin RM, Schneider W. Controlled and automatic human information processing: II. Perceptual learning, automatic attending and a general theory. Psychological Review. 1977; 84(2):127-190.

Smith SM, Vela E. Environmental context-dependent memory: A review and meta-analysis. Psychonomic Bulletin \& Review. 2001; 8(2):203-220. [PubMed: 11495110]

Theeuwes J. Cross-dimensional perceptual selectivity. Perception \& Psychophysics. 1991; 50(2):184193. [PubMed: 1945740] 
Theeuwes J. Perceptual selectivity for color and form. Perception \& Psychophysics. 1992; 51(6):599_ 606. [PubMed: 1620571]

Thompson C, Underwood G, Crundall D. Previous attentional set can induce an attentional blink with task-irrelevant initial targets. Quarterly Journal of Experimental Psychology. 2007; 60(12):16031609.

Todd S, Kramer AF. Attentional misguidance in visual search. Perception \& Psychophysics. 1994; 56(2):198-210. [PubMed: 7971120]

Tseng C-H, Gobell JL, Sperling G. Long-lasting sensitization to a given colour after visual search. Nature. 2004; 428:657-660. [PubMed: 15071596]

Turatto M, Galfano G. Attentional capture by color without any relevant attentional set. Perception \& Psychophysics. 2001; 63(2):286-297. [PubMed: 11281103]

Vickery TJ, King LW, Jiang Y. Setting up the target template in visual search. Journal of Vision. 2005; 5(1):81-92. [PubMed: 15831069]

Waszak F, Hommel B, Allport A. Task-switching and long-term priming: Role of episodic stimulustask bindings in task-shift costs. Cognitive Psychology. 2003; 46:361-413. [PubMed: 12809680]

Wolfe JM, Horowitz TS. What attributes guide the deployment of visual attention and how do they do it? Nature Reviews Neuroscience. 2004; 5(6):495-501. [PubMed: 15152199]

Wolfe JM, Horowitz TS, Kenner N, Hyle M, Vasan N. How fast can you change your mind? The speed of top-down guidance in visual search. Vision Research. 2004; 44(12):1411-1426.

[PubMed: 15066400] 
Training Session

320 Trials
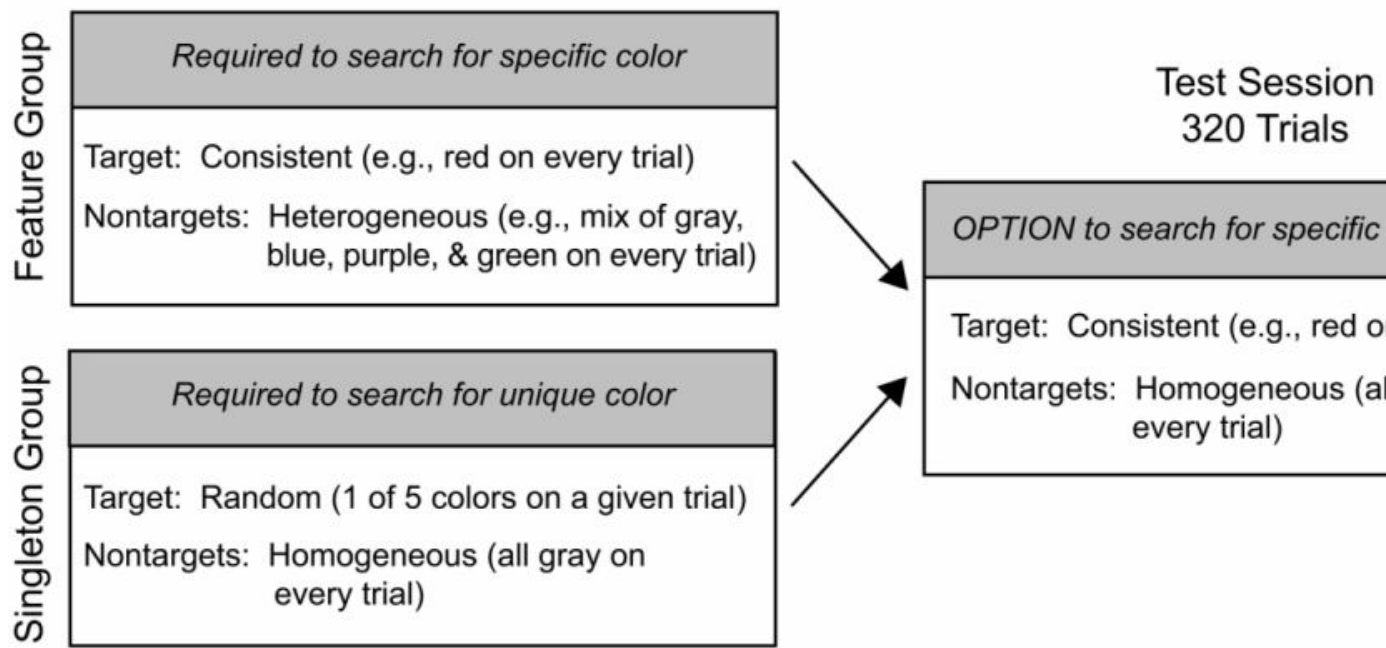

OPTION to search for specific or unique color

Target: Consistent (e.g., red on every trial)

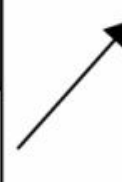

Nontargets: Homogeneous (all gray on every trial)

\section{Figure 1.}

Overview of training and test session conditions for Feature and Singleton groups used by Leber \& Egeth (2006) and in Experiment 1. 


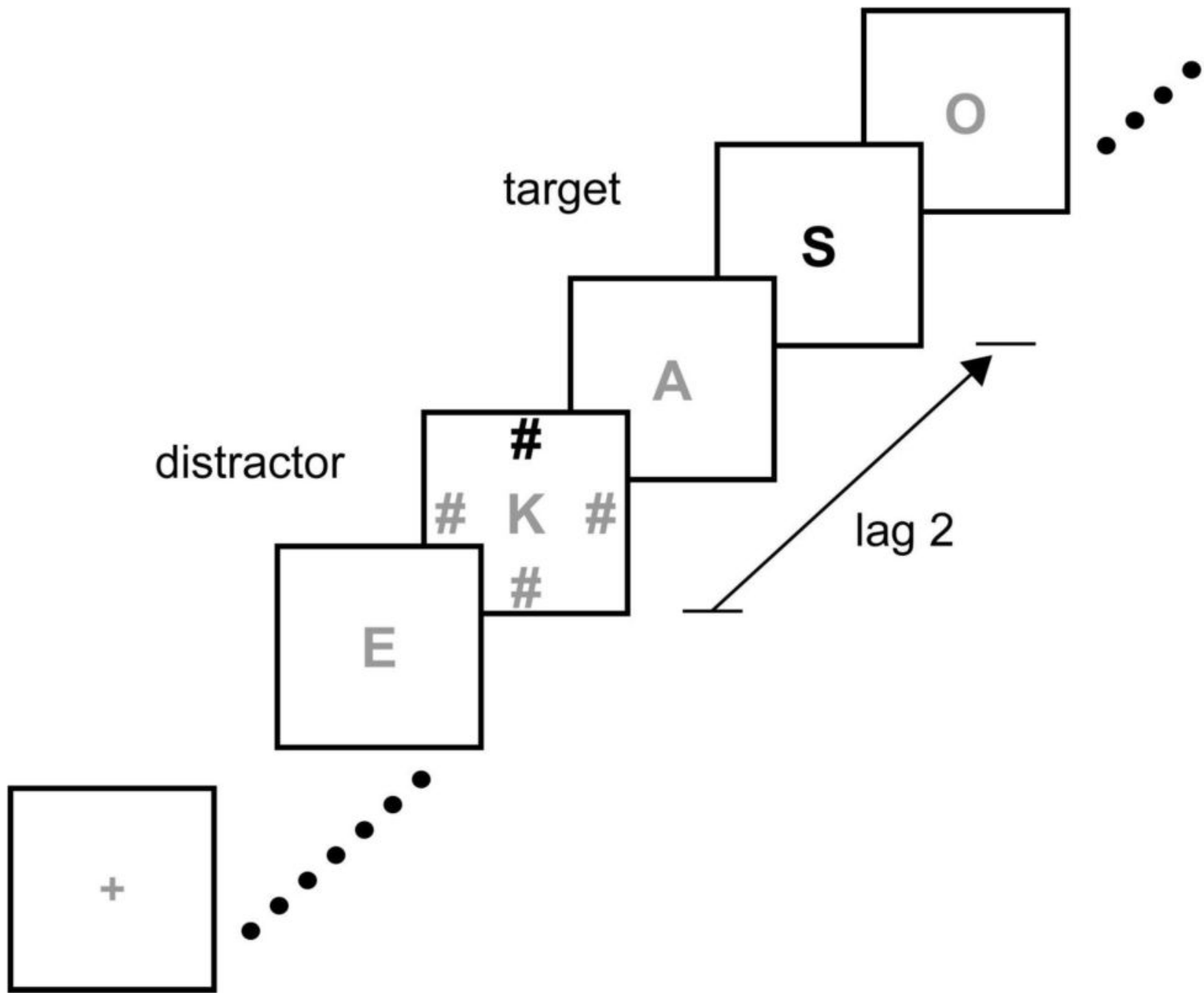

Figure 2.

Representation of trial sequences used by Folk et al. (2002) and in Experiment 1. In this example, a distractor display containing a color singleton appears at a lag of two items (approx. $200 \mathrm{~ms}$ ) prior to the target. Black characters were colored red or green (see text for details). 
1 Day

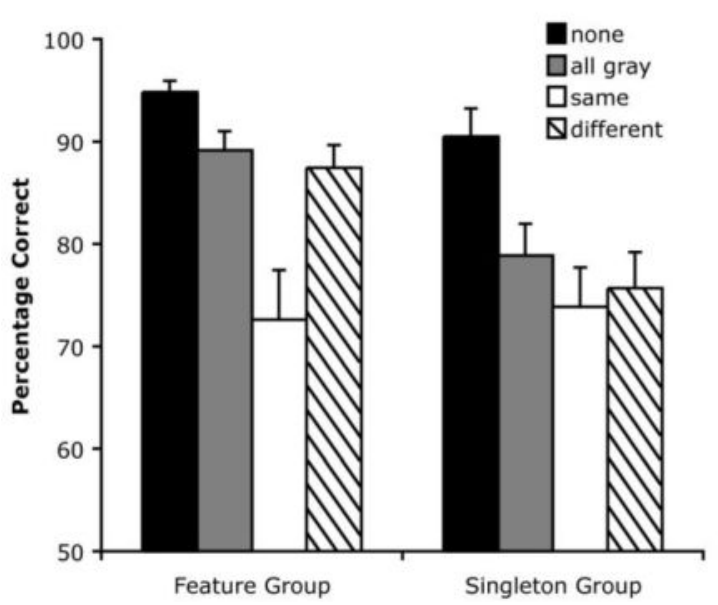

1 Week

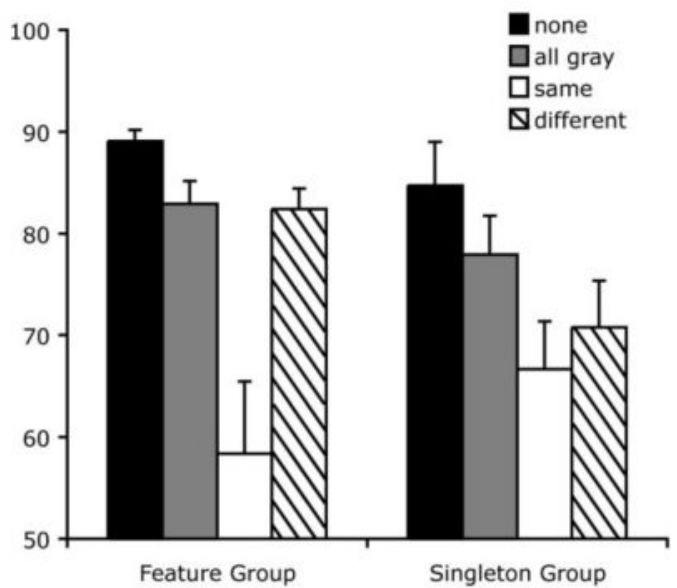

Figure 3.

Performance in the Test session of Experiment 1. Shown are mean accuracy scores (percentage correct) for the feature and singleton groups as a function of distractor type, with one day (left panel) and one week (right panel) delays following training. Error bars indicate standard error of the mean. 


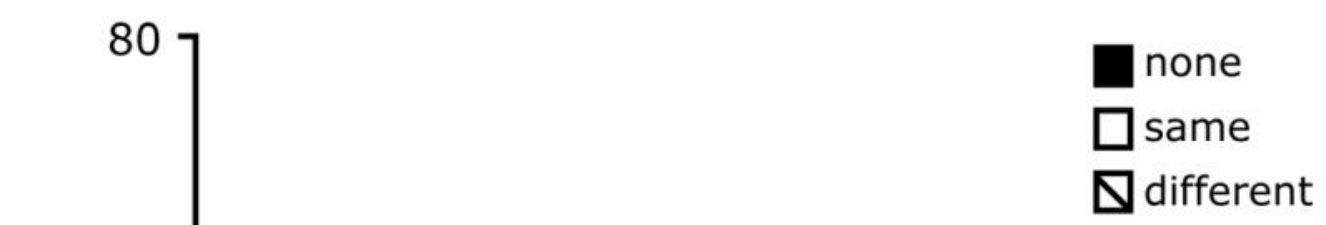

Figure 4.

Performance in the Test phase of Experiment 2. 


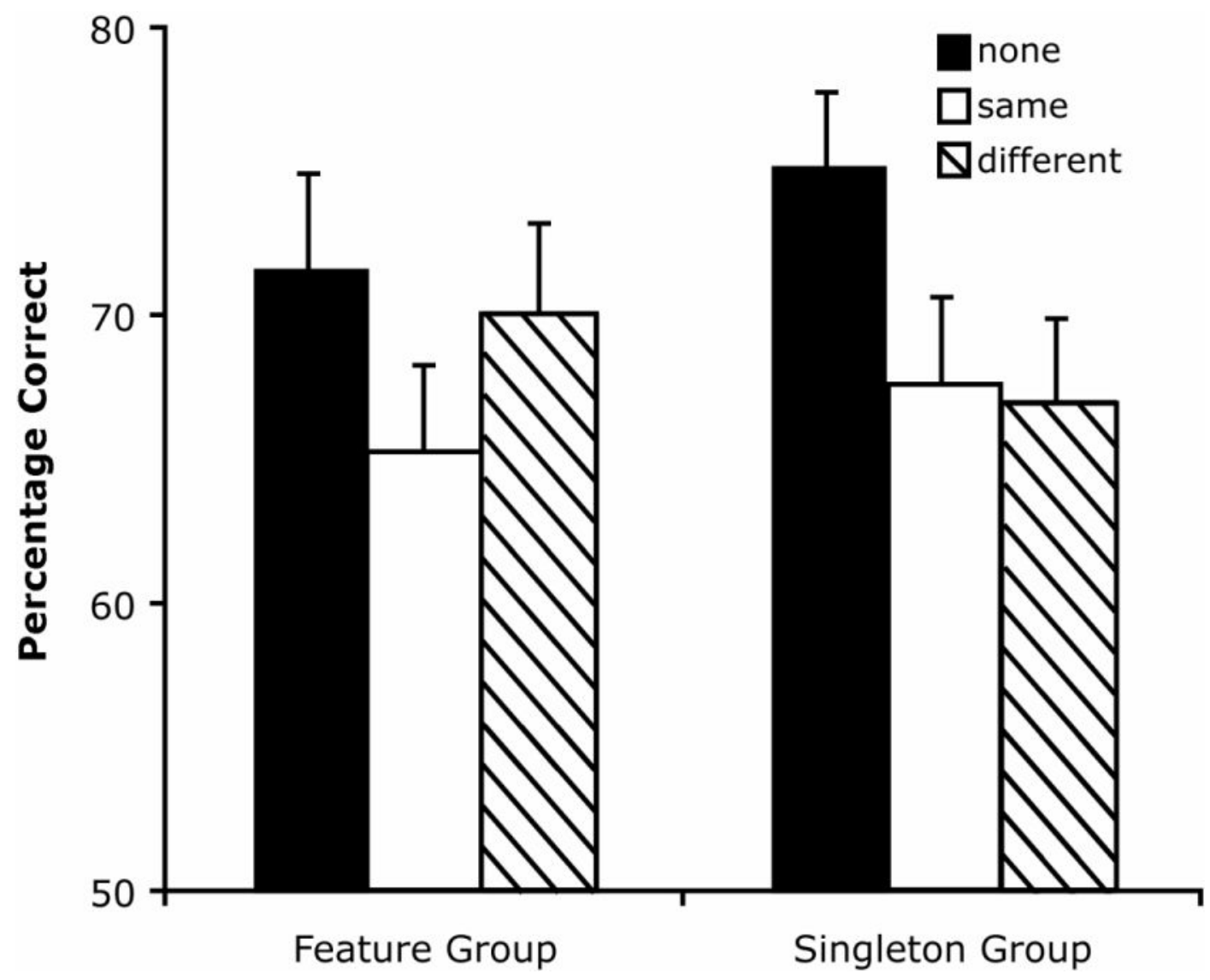

Figure 5.

Performance in the Test phase of Experiment 3. 